\title{
A BIBLIOMETRIC ANALYSIS ON THE DISSEMINATION OF COVID -19 VACCINE MISINFORMATION ON SOCIAL MEDIA
}

\author{
Renuka Mahajan \\ Assistant Professor, Jaipuria Institute of Management, Noida \\ rmahajan@jaipuria.ac.in \\ Pragya Gupta \\ Assistant Professor, Jaipuria Institute of Management, Noida \\ pragya.g@jaipuria.ac.in
}

\begin{abstract}
In recent years, COVID-19 vaccine-related issues and viewpoints have aroused significant anxiety and concern. Several research studies are extracting, tracking, and evaluating prevalent public opinions on social media and making efforts to curb the misinformation spread. But, there is still a large audience that perceives vaccination as a threat, which in turn reduces our ability to fight effectively against the pandemic. This bibliometric study aims to explore the distribution of capabilities of researchers, institutions, and countries, research themes, and frontiers of Covid-19 vaccine-related misinformation trending on social media since the rollout of these vaccines. The Scopus online database was used for analysis. Excel 2016 and VOSViewer (version 1.6.17) software were used to report the visualizations of infodemic literature on COVID Vaccine on social media. Annual publications, top contributing authors, top-cited journals and author affiliation, leading subject areas, the top country in publication, and keyword network were among the key findings. Future researchers can use these findings to create a baseline before studying Covid-19 vaccine misinformation on social media. Furthermore, it may help in compiling crucial knowledge, trends, and lessons from existing researches to provide useful insights to handle similar phenomena in the future.
\end{abstract}

Keywords: COVID-19 vaccine; social media; vaccine hesitancy; vaccine efficacy; public opinions; infodemic; misinformation

\section{INTRODUCTION}

The World Health Organization (WHO, 2020) labeled COVID-19 outbreak as a pandemic during early March, of that year. Alongside preventive measures like masks and social distancing, the development of vaccines proved to be a vital tool in fighting against the pandemic (Loomba et al, 2021). While several vaccines exist for various other diseases, the kind of debate that took the world by storm on vaccine efficacy had never been seen before.

Perhaps it was because of the unprecedented rate at which vaccine development and trials were undertaken in various countries (Mahajan et al, 2022). There was an urgency to develop a suitable COVID-19 vaccine to control widespread deadly disease resulting in a high death rate, which had not been seen since the 1900s (Centres for Disease Control and Prevention, 2019).
Furthermore, given the evolving nature of the virus and its variants, it's uncertain how long vaccination-induced immunity will last. Despite frenzy around the disbeliefs related to the COVID-19 vaccine, vaccination remains our most effective tool to counter this pandemic. To offer herd immunity from getting infected, at least 55 percent of the country's population must be vaccinated, with estimations ranging as high as 85 percent depending on the infection rate and country (Kwok et al, 2020; Sanche et al, 2020). Given the participatory nature of social media, social interactions on these platforms are one of the most effective ways to influence the sentiments of the masses. This is especially true when people discuss COVID-19 issues and related remedies, and use them as a coping technique during lockdowns and pandemic-related situations (Praveen et al, 2021). 
Social media is also a reliable source for researchers for extracting, tracking, and evaluating prevalent public opinions, personal experiences, and identifying health information needs (Sinnenberg, 2017). However, it is easy to intersperse online discourses on social media with false, misleading, or inaccurate information, most of which tend to propagate faster than credible information (Muric et al, 2021). While the number of vaccines approved for use by WHO has risen extraordinarily over the last year (Tregoning et al., 2021), yet the inoculation rate is still falling short, and communication approaches, especially 'social media' plays a major role in this. With all kinds of opinions from people all over the world, some of which fake their identities, the spread of misinformation regarding vaccination is rampant. It could be fueled by religious and philosophical reasons along with personal beliefs and conspiracy theories (McClure et al, 2017).

The complexity of social media dissemination increases as it not only entails genuine humanrun accounts indulging in anti-vaccine sentiments but also has to account for the role of inorganic users like bots and trolls that is increasingly being used to disseminate information regarding the COVID-19 vaccine on social media (Broniatowski et al., 2018). With so many opposing and diverse views; it is hard to discern what to believe and thus, people start questioning everything that they trusted before, including vaccines (Kricorian, Civen, \& Equils, 2021).

By instilling skepticism and cynicism about new vaccinations, such content can intensify pre-existing worries and fears, restricting public acceptance of COVID-19 vaccines. This could drastically jeopardize the herd immunity objectives against the deadly virus, as desired by public health experts worldwide. Previous research indicates that people all across the world have been affected by the recent social media buzz about the COVID-19 vaccination (Quinn et al., 2020; Bogart et al., 2020). Hence, this calls for careful monitoring and reporting of any inaccurate or false information regarding vaccination that is found trending on social media.

The ongoing pandemic is significant because it offers an opportunity to study all related parameters apart from the fact that it is the first time that a pandemic is being documented, argued, and examined in realtime by the scientific community as they gain more experience (Boetto et al, 2021). One of the most popular pandemic research topics is examining the public opinion regarding the Covid-19 vaccination (Thunstrom, 2020; Fu, 2020). Compared to the traditional medium, anti-vaccination messages may spread more easily and swiftly on social media, without being subjected to editorial curation or scientific vetting, that too anonymously (Meleo-Erwin, 2017; Massey, 2018).

The social media users' perceptions can thus grow around anti-vaccine sentiments (Burki, 2019). Anti-vaccination propaganda can be amplified by social media and hence COVID vaccine misinformation through the lens of 'social media' is studied extensively by several researchers (Roozenbeek, 2020) to have a better understanding of what vaccine opponents are saying, how their ideas are spreading, and who is primarily responsible for it.

A bibliometric study is thus crucial to compile more knowledge, trends, and lessons learned from such emerging research areas, as the pandemic is far from getting over and even if it does, we need to be prepared for similar future catastrophes. However, to the best of the authors' knowledge, no similar bibliometric analysis on the role of social media for COVID vaccine misinformation has been published so far. Hence, the current study fills the gap. The aim of the current bibliometric study is to present the analysis of the key themes and topics in this field.

The current study attempts to analyze the publication trend of research in the area, most cited journals, journals with maximum publications, active countries and organizations working in the area, frequently used keywords, co-authorship, etc. The findings of the study would provide future directions in keeping with the World Health Organization's (WHO) vision for action in managing the pertinent concern of widespread infodemics within the pandemic crisis.

\section{METHODS}

The Scopus online database was used for analysis as it contains the majority of quality 
research papers along with in-built analysis capabilities for providing representative figures. Besides, the Scopus search results could be directly exported to VOSViewer software for further analysis. The details of the publications that met the criteria like the year of publication, top nations carrying out this research, authors, source title, research title, language, institutional affiliation, keywords, type of document, abstract, and number of citations per article were all saved into CSV format. The date of the retrieval was $2^{\text {nd }}$ December 2021. The authors utilized VOSviewer (version 1.6.17) to analyze the Coauthorship, Co-occurrence, and themes. The search query carefully selected by the authors was based on all possible metaphors utilized in the literature.

The initial search included the following search terms: 'social media', 'social networking, 'social listening', 'misinformation', 'infodemic', 'disinformation', 'fake news', 'misconception', 'Conspiracies', 'Conspiracy', 'COVID', 'COVID19', 'coronavirus', 'COVID-19', 'pandemic', 'coronavirus disease 2019', '2019n COV', 'SARS-Cov-2', 'novel coronavirus', 'Severe Acute Respiratory Syndrome CoronaVirus 2', 'SARS-CoV-2', 'nCoV-2019', 'vaccine', 'vaccine hesitancy', 'vaccine safety', 'vaccine efficacy', 'vaccine confidence', 'vaccine development'.

\section{RESULTS}

Our search yielded 227 studies that widely contained data and material pertaining to COVID-19 vaccine and/or severe acute respiratory syndrome coronavirus 2 (SARS$\mathrm{CoV}-2)$ vaccine or coronavirus vaccine and social media and misinformation or an infodemic that appeared between December 2019 and December 2021. Those articles that were from languages other than English were excluded due to a lack of translation resources.

Two papers belonging to the Measles vaccine hesitancy was further excluded as it did not pertain to the current research scope. There were no limitations on the current status of the publication, which means articles in the press were also included in the study. Thus, after removing duplicates and articles not related to the topic, 218 publications were finally selected for inclusion in our analysis. (Figure 1).

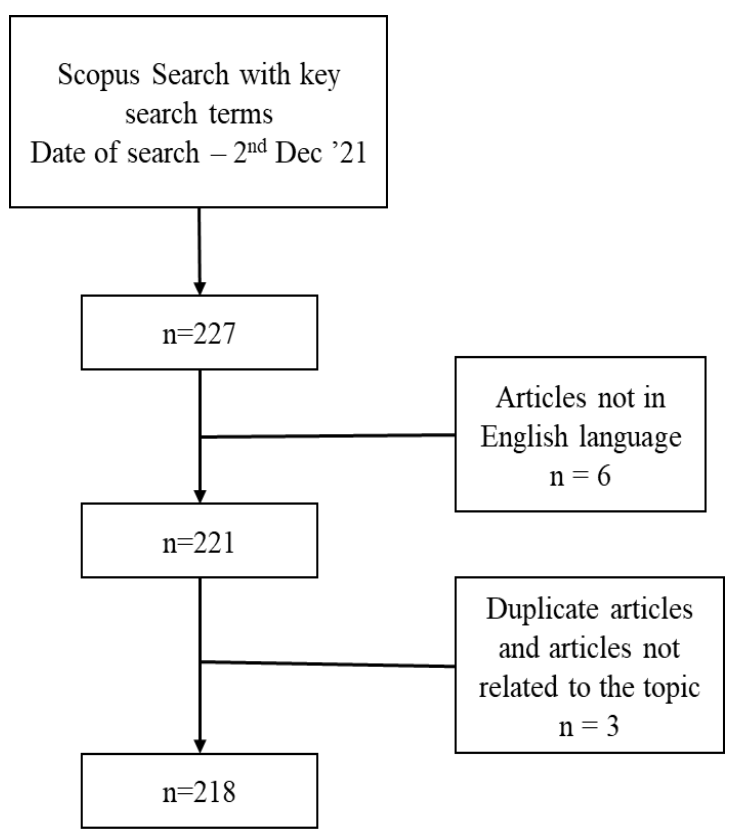

Figure 1: Search criterion and extraction of data

\subsection{Distribution trend of annual publication output}

From 50 publications in 2020, the research publication increased to 167 publications in 2021 as shown in Table 1. One paper was also found for 2022. The table also reveals the total number of citations year-wise. Even before the vaccines were rolled out at the end of December 2020, anti-vaccine sentiments were rife on social media as is evident from the researches (Welle, 2021).

Table 1: Publication Output

\begin{tabular}{|c|c|c|}
\hline Year & $\begin{array}{c}\text { Total Number of } \\
\text { Publications }\end{array}$ & $\begin{array}{c}\text { Count of } \\
\text { Citations }\end{array}$ \\
\hline 2022 & 1 & 6 \\
\hline 2021 & 167 & 779 \\
\hline 2020 & 50 & 1858 \\
\hline
\end{tabular}

\subsection{Distribution of publications among most} active countries

The most active countries having maximum publications on social media misinformation related to COVID-19 vaccine are listed in Table 2. It shows that the US published the maximum number of papers i.e., 91 papers, followed by the UK with a count of 28 publications which is then followed by Australia (14), Canada (13), Pakistan (11), China (10), Italy/ Sweden (9 each), France/ Jordan/ Spain (8 each), India/ Saudi Arabia (7 each) and finally Malaysia/ Poland (6 each). 
Table 2: Most active countries in terms of publications

\begin{tabular}{|c|c|c|c|}
\hline Ranking & Country & $\begin{array}{c}\text { Total } \\
\text { Number of } \\
\text { Publications }\end{array}$ & $\begin{array}{c}\text { Citation } \\
\text { count }\end{array}$ \\
\hline 1 & US & 91 & 2660 \\
\hline 2 & UK & 28 & 593 \\
\hline 3 & Australia & 14 & 124 \\
\hline 4 & Canada & 13 & 329 \\
\hline 5 & Pakistan & 11 & 96 \\
\hline 6 & China & 10 & 97 \\
\hline \multirow{2}{*}{7} & Italy & 9 & 203 \\
\cline { 2 - 4 } & Sweden & 9 & 528 \\
\hline \multirow{2}{*}{8} & France & 8 & 343 \\
\cline { 2 - 4 } & Jordan & 8 & 169 \\
\cline { 2 - 4 } & Spain & 8 & 36 \\
\hline \multirow{2}{*}{9} & India & 7 & 102 \\
\cline { 2 - 4 } & Saudi Arabia & 7 & 104 \\
\hline \multirow{2}{*}{10} & Malaysia & 6 & 64 \\
\cline { 2 - 4 } & Poland & 6 & 72 \\
\hline
\end{tabular}

3.3 Distribution of top journals in terms of the citations and number of publications

It is observed that a total of 132 journals published articles on social media misinformation on COVID 19 vaccine. Figure 2 (A) depicts the top 10 journals with the most cited articles. The maximum number of citations for the Journal of Medical Internet Research with 469 citations followed by Journal of Travel Medicine (327), Human Vaccine and Immunotherapeutics (172), International Journal of Environmental
Research and Public health (153), Social Science and Medicine (144), Vaccines (144), European Journal of Epidemiology (105), BMJ Global Health (105), Vaccine (96) and PLoS ONE (81) journals.

The snapshot of journals with most number of published articles is shown in figure 2(B). The maximum number of articles were published by the International Journal of Environmental Research and Public Health (14), followed by Journal of Medical Internet Research (13), Vaccines (13), Human Vaccines and Immunotherapeutics (9), PLoS one (8), JMIR Public health and Surveillance (5), Science and Vaccine with 4 publications and finally Nature Immunology, Public Health, Pan African Medical Journal, Science, Big data and Society, The BMJ and with 3 publications each.

\subsection{Distribution of articles based on the subject area}

Figure 3 shows the top position subject areas with the maximum number of articles on social media misinformation on the COVID-19 vaccine. 'Medicine' is ranked one with 157 publications followed by 'Immunology and Microbiology' with 38 publications. 'Social Sciences' and 'Pharmacology, Toxicology and Pharmaceutics' are at rank three with 30 publications each. Then, the top research areas were Computer Science (23), Multidisciplinary (16), Environmental Science (15),

\section{Top Journals with maximum citations}



Figure 2 (A): Top Journals with maximum citations 


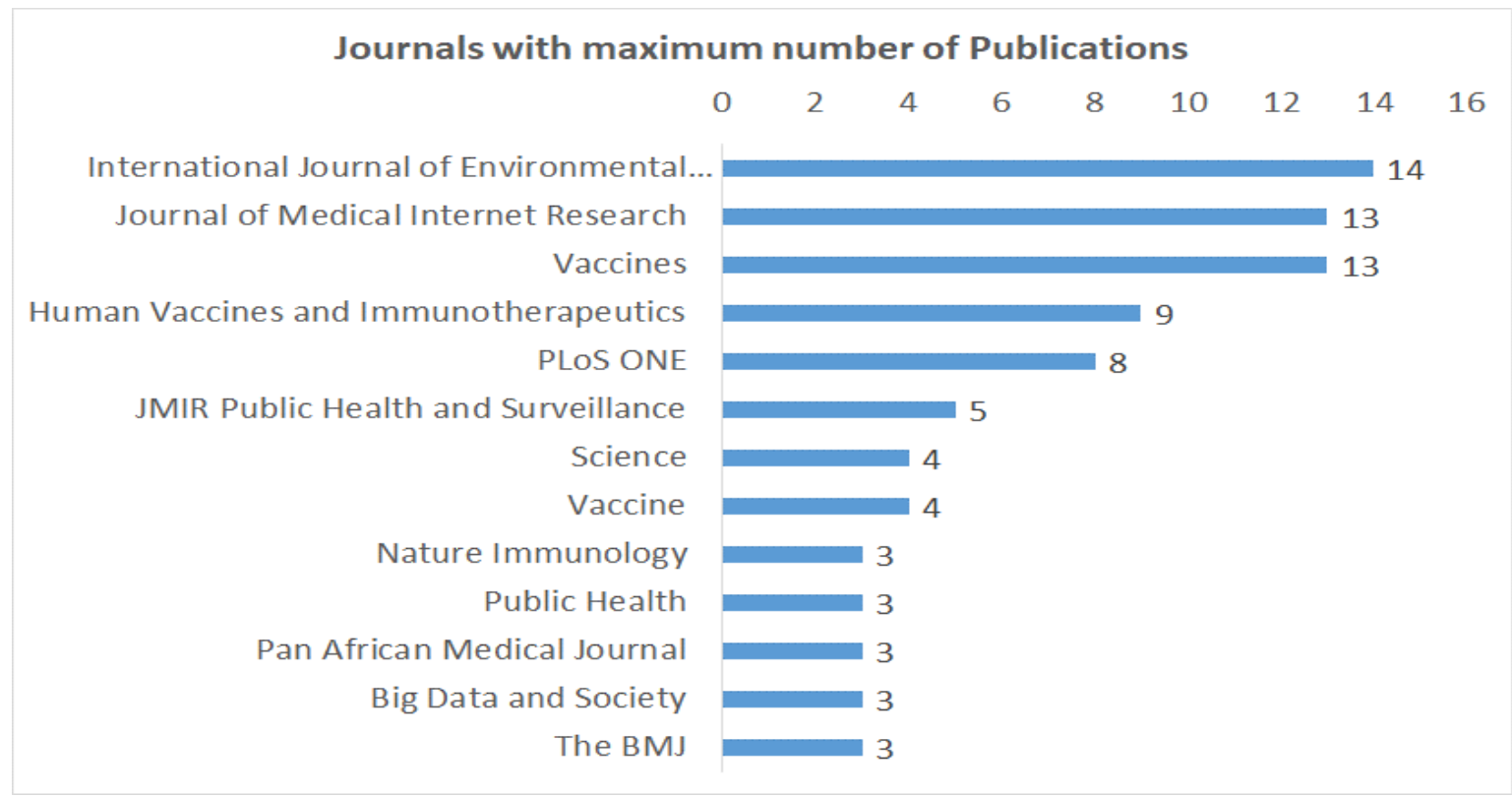

Figure 2(B): Top Journals with maximum publications

Biochemistry, Genetics and Molecular Biology (12), Nursing (10), Engineering (8), Health Professions (6), Decision Sciences (6), Psychology (5), Arts and Humanities (4), Veterinary (4), Mathematics (3), Neuroscience (3), Energy (2), Business, Management and Accounting (2), Materials Science (1) and Earth and Planetary Sciences (1).

\subsection{Distribution of articles based on different affiliations}

'Harvard Medical School' has the maximum number of publications with 10 articles published, followed by 'Harvard University' with 8 publications and "The University of Jordan' with 6 publications. Jordan University Hospital, London School of Hygiene \&



Figure 3: Publications based on the subject area 
Tropical Medicine, University of Toronto and The George Washington University hold the fourth position with 5 publications each. The next position is held jointly by Columbia University, Lunds Universitet, University of Oxford, University of Pennsylvania, and Universiti Sains Malaysia with 4 publications each. Finally, School of Medicine, Harvard T.H. Chan School of Public Health, Milken Institute School of Public Health, University of California, San Francisco, University of Connecticut, University of Toronto, William Paterson University, Brown University, City University of New York, Google LLC and Johns Hopkins Bloomberg School of Public Health followed with 3 publications as seen in figure 4 .

\section{Bibliometric Analysis of themes and trend topics}

Figure 5 (A) revealed four themes of COVID19 vaccine misinformation on social media were extracted. The red cluster involved the 'awareness and preparedness towards handling COVID 19 outbreak'. The green cluster depicted 'information and communication strategies'. The blue cluster represented 'demographic cohorts in coronavirus studies'. The yellow cluster involved the 'anti-vaccine disposition'. Figure 5 (B) represents the network mapping of the trending topics according to the author keywords used in the researches since December 2019 till date, according to publication timelines.

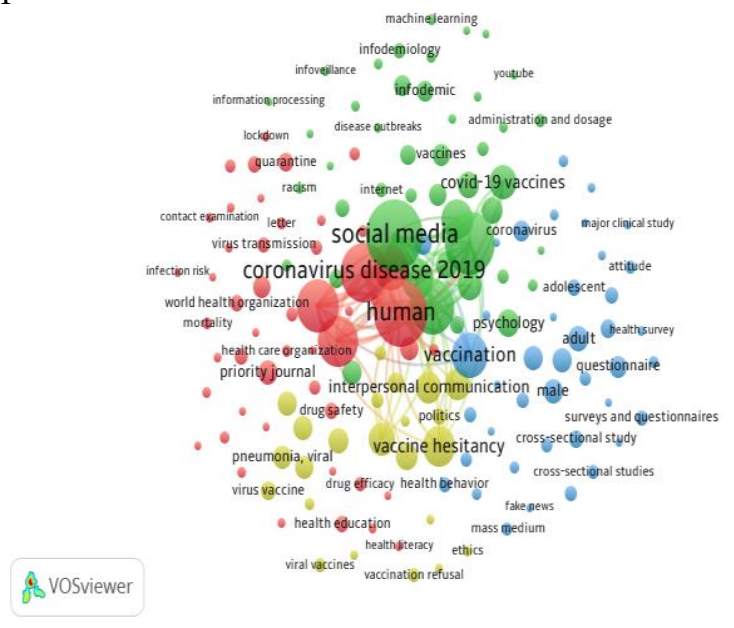

Figure 5 (A): Analysis of the distribution of themes

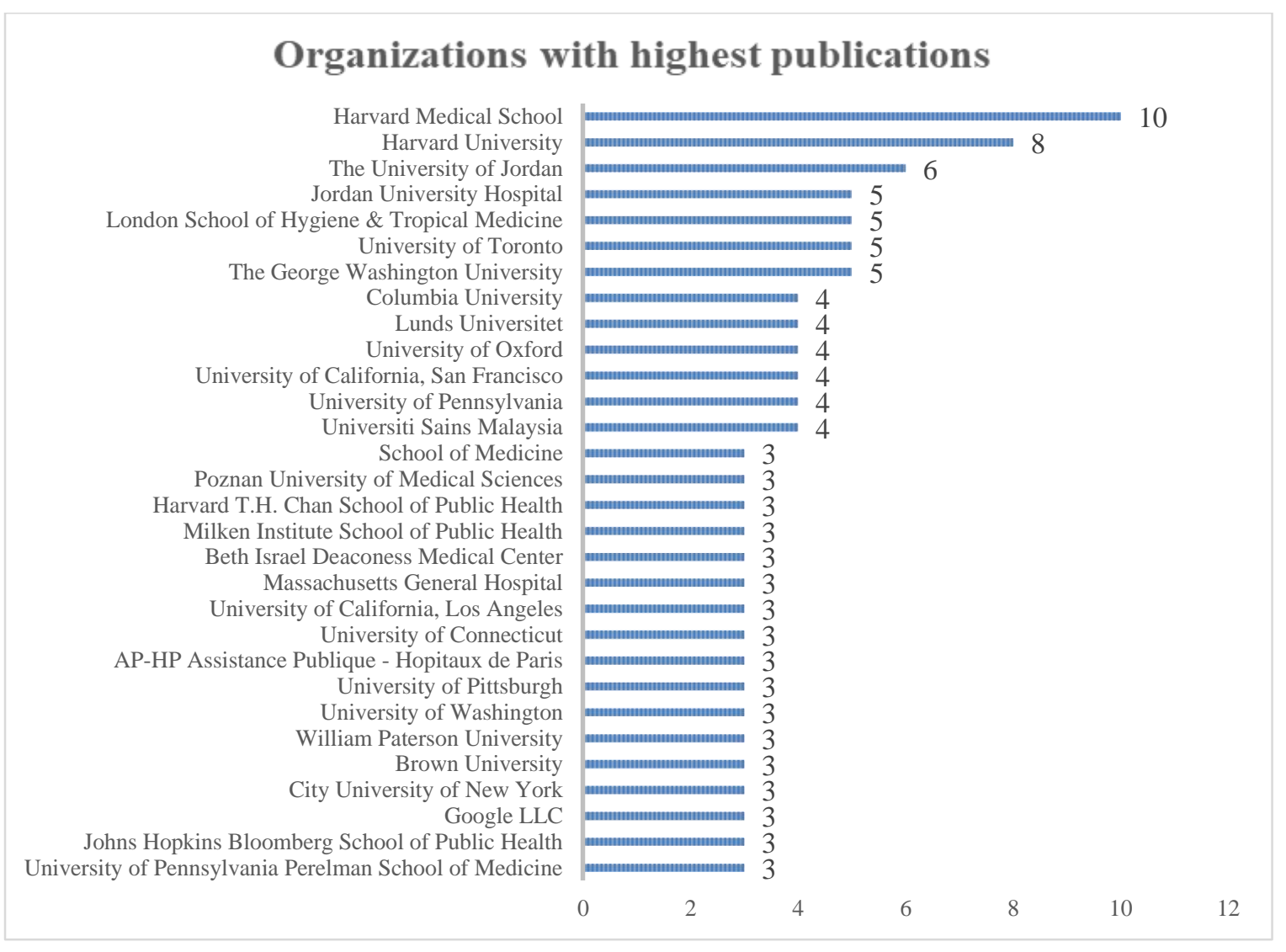

Figure 4: Organizations with maximum publications 


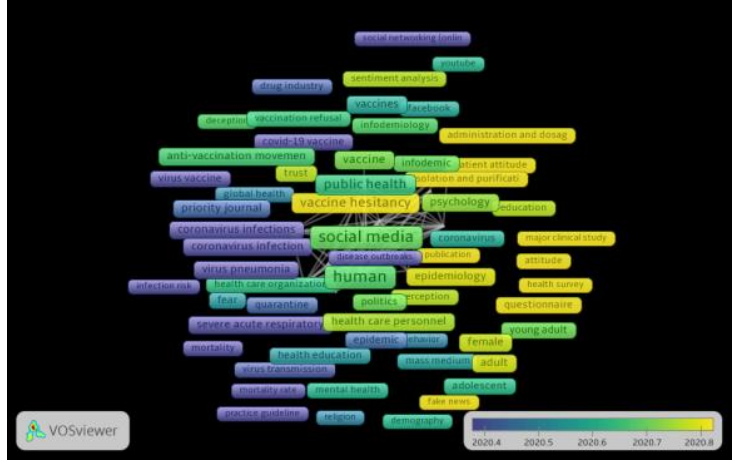

Figure 5 (B): Network mapping of trends according to publication timelines
3.7 Bibliometric analysis of author keywords As indicated in figure 6 (A), keywords given by authors of the work with a minimum occurrence of 5 were recruited for the final keyword analysis. Out of 420 keywords only 28 met the required threshold. The overall strength of the co-occurrence of links with other keywords was determined for each of these 28 keywords. The authors chose the keywords with the most total link strength. The size of the nodes represents the frequency with which they occur. The co-occurrence of the nodes is shown by the curves between them. It was found that the greater the

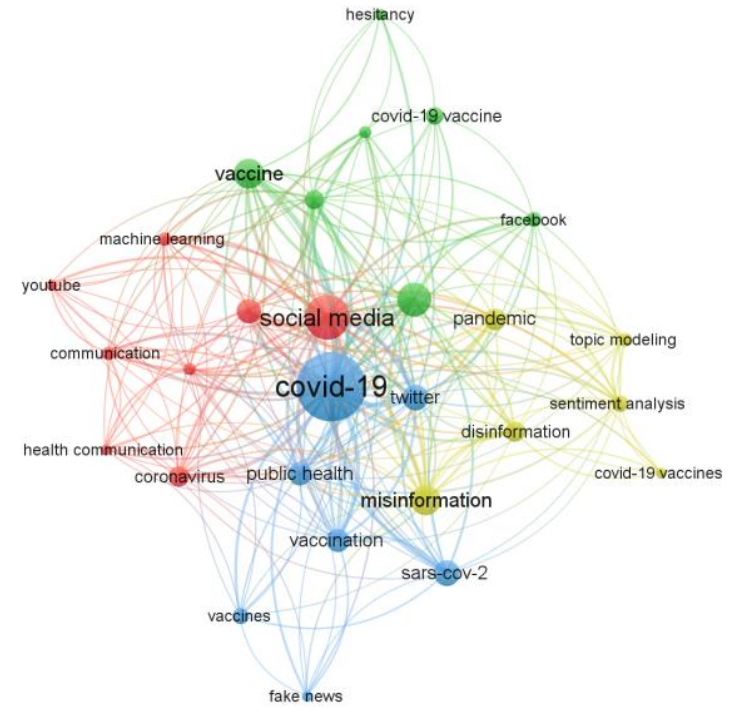

Figure 6 (A): Analysis of co-occurrence of keywords

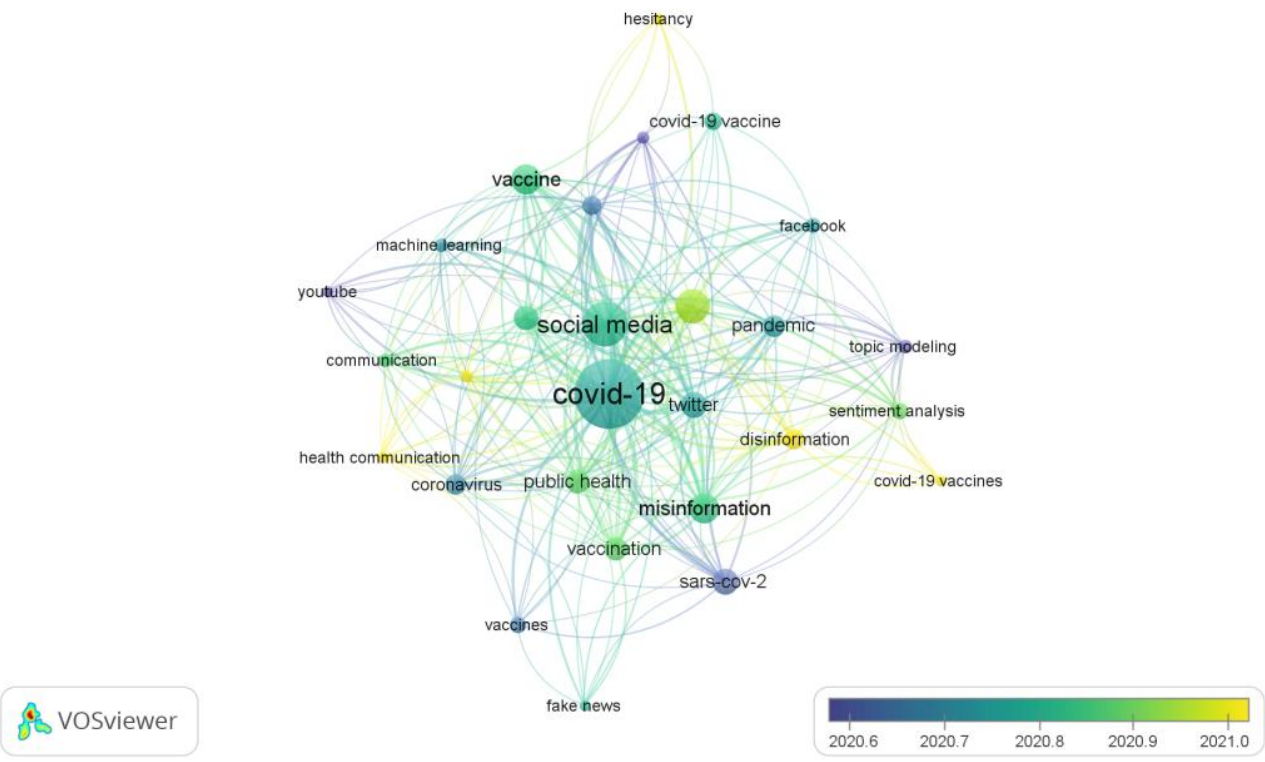

Figure 6 (B): Analysis of co-occurrence of keywords according to publication timelines 
frequency of co-occurrences of two terms, the shorter the distance between two nodes. The font size denotes the frequency with which the event occurs. The keywords with maximum frequency of occurrences included 'COVID-19' (total link strength 260) and 'social media' (total link strength 179). 'Twitter', 'vaccine hesitancy' and 'misinformation' were the next three most occurring keywords and the total link strength of each was found to be more than 80. Figure 6 (B) displays the cooccurrence of keywords according to publication timelines. forensic medicine, Jordan University Hospital partnered with the School of Medicine, The University of Jordan, and Lund University, Sweden to publish 4 papers with 159 citations. Cell therapy center, the University of Jordan, and the School of dentistry, the University of Jordan were other important partners to publish three papers together with 158 citations.

The analysis of country-wise co-authorship distribution reveals that the highest number of publications is from the US and the total link

gifford d. vassar m. gokhale s.s.

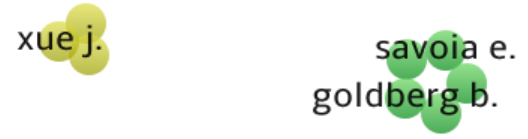

hut.

jamieson k.h.

Iarson h.j.
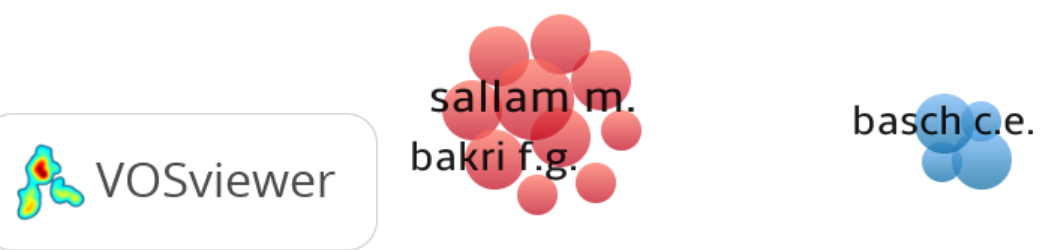

Figure 7 (A): Bibliometric analysis of the co-authorship map of authors

\subsection{Bibliometric analysis of co-authorship}

This topic has a total of 919 authors that have contributed to its publishing. The analysis of 36 such authors, who published a minimum of 2 articles has been presented in figure 7(A). At the top position in this list was M. Sallam from Jordan University Hospital, who has 4 papers that mostly focussed on the COVID-19 vaccine hesitancy and conspiracy beliefs. The main collaborators with him are D. Dababseh from Jordan University Hospital and, F.G. Bakri \& A. Yaseen from The University of Jordan, Amman. The total link strength was found to be 24 .

According to the domestic and international literature search, 671 organizations have published related articles, with 20 of them having more than two. As clear from figure 7 (B), the Department of clinical laboratories \& strength is 49 with 86 publications and 1072 citations; followed by UK's total link strength is 33 with 26 publications and 551 citations (Figure $7(\mathrm{C})$ ).

\section{DISCUSSIONS AND CONCLUSION}

In this study, we looked at 218 publications about COVID-19 vaccine misinformation on social media that were indexed in the Scopus database. The number of scientific articles in 2021 have risen to 167 from 50 publications in 2020. The United States had contributed the most within this important field. The topmost journals in terms of most-cited publications were Journal of Medical Internet Research, followed by Journal of Travel Medicine, Human Vaccine and Immunotherapeutics, International Journal of Environmental research and Public Health, Social Science and Medicine, Vaccines, BMJ Global Health, 
department of public health, w

department of health, behavior

department of clinical laborat

department of psychology, univ

\section{S VOSviewer}

department of infectious disea

Figure 7 (B): Bibliometric analysis of the co-authorship map of organizations

pakistan

china

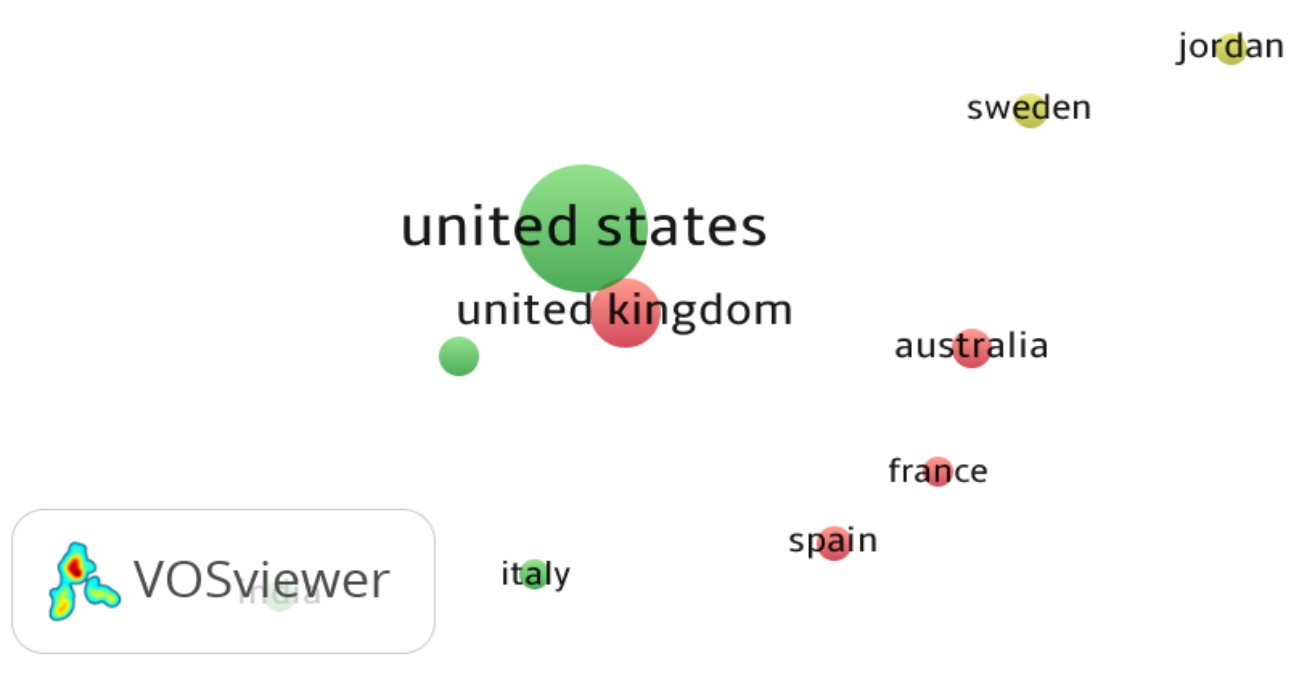

Figure 7(C): Bibliometric analysis of the co-authorship map of countries

European Journal of Epidemiology, Vaccine and PLoS ONE journals. The journals with maximum number of publications on the topic included International Journal of Environmental research and Public Health, followed by Journal of Medical Internet Research, Vaccines, Human Vaccine and Immunotherapeutics, PLoS ONE, JMIR Public Health and Surveillance, Science, Vaccine, Nature Immunology, Public Health, Pan
African Medical Journal, Big Data and Society and The British Medical Journal (BMJ).

Medicine, Immunology and Microbiology, Pharmacology, Toxicology and Pharmaceutics and Social sciences were the top subject areas. Leading organizations with maximum number of publications included Harvard Medical School, followed by Harvard University and The University of Jordan. The 
published literature includes the following themes: 'awareness and preparedness towards handling COVID 19 outbreak', 'information and communication strategies', 'demographic cohorts in coronavirus studies' and 'antivaccine disposition'. The keyword "COVID19 " was found to have the strongest link to "social media", followed by keywords such as 'Twitter', 'vaccine hesitancy' and 'misinformation' as the most frequently used keywords. Author M. Sallam of Jordan University Hospital was recognized to be the top author, with four publications already published on COVID-19 vaccine hesitancy and conspiracy theories. Authors D. Dababseh of Jordan University Hospital, F.G. Bakri, and A. Yaseen of the University of Jordan's School of Medicine in Amman were identified as his principal collaborators. Out of all the organizations, Jordan University Hospital's Department of Clinical Laboratories and Forensic Medicine has the most papers and they have collaborated with the University of Jordan's School of Medicine and Lund University in Sweden for maximum publications and citations as the results depict. Timeline indicator bar with yellow color indicates that the studies are recently focusing on 'COVID-19 vaccine', 'human experiments', 'disinformation', 'drug efficacy', 'vaccine hesitancy' which may be considered to be hotspots in this field in future.

COVID-19 vaccine-related misinformation on social media has sparked widespread anxiety and concern in recent months. As a result, several research studies conducted in this area are trying to debunk the myths and promote genuine content from public health agencies; otherwise, the vaccination movement is likely to suffer. The issue of COVID-19 vaccine hesitancy varies across countries and geographies, pre and post-vaccination or between different socio-demographic groups and with varying timelines, as more advances in treatments are gradually discovered. Several pieces of research investigate the changing trend of public willingness to accept the vaccine, influenced by information (or misinformation) trending on social media platforms (Loomba et al., 2021). Against this background, a bibliometric study of these articles may point to important themes and future research directions. The findings have implications for anyone (scientist and public health experts) on the internet and social media to understand the key issues surrounding the false or misinformation on social media that adds to COVID vaccine hesitancy. Based on the findings, one can carry out evidence-based educational campaigns on creating awareness and communication to successfully dispel any misinformation about the safety of further treatment advances as discovered over time.

\section{LIMITATIONS AND FUTURE SCOPE}

The current bibliometric study utilized the research papers that conducted their research on real-world data set from social media. The social media study can include the views of only online users and exclude the views of people accessing other traditional media outlets (such as radio or community events). As a result, it may fall short in identifying information gaps leading to COVID-19 vaccine-related rumors originating at other places (including religious leaders), where the people, especially those without access to technology, look for health information. Hence, future bibliometric analyses should compare the studies that use offline interventions to survey how people are responding to and interpreting vaccine-related communication. Future studies can explore how different authors' regions and coauthor networks affect their research perspectives. Besides, a comparison can be made on the different research methods used so far on misinformation. Furthermore, non-English language articles were excluded from the analysis due to the lack of translation resources need to judge their relevancy in this research. Since the COVID-19 outbreak was initially detected in Wuhan in China, research publications in Chinese language could be useful and may be included in future studies.

\section{REFERENCES}

Boetto, E., Fantini, M. P., Gangemi, A., Golinelli, D., Greco, M., Nuzzolese, A. G., ... \& Rallo, F. (2021). Using altmetrics for detecting impactful research in quasizero-day time-windows: the case of COVID-19. Scientometrics, 1-27.

Broniatowski, D. A., Jamison, A. M., Qi, S., AlKulaib, L., Chen, T., Benton, A., ... \& Dredze, M. (2018). Weaponized health communication: Twitter bots and Russian trolls amplify the vaccine 
debate. American journal of public health, Vol. 108, No. 10, pp.1378-1384.

Burki, T. (2019). Vaccine misinformation and social media. The Lancet Digit. Heal. 1(6), e256-e259,

https://doi.org/10.1016/S25897500(19)30136-0

Centres for Disease Control and Prevention (March 20, 2019). 1918 Pandemic (H1N1 virus) Available at: https://www.cdc.gov/flu/pandemicresources/1918-pandemic-h1n1.html (Accessed 12th October 2021)

Johnson, N. F., Velásquez, N., Restrepo, N. J., Leahy, R., Gabriel, N., El Oud, S., ... \& Lupu, Y. (2020). The online competition between pro-and anti-vaccination views. Nature, 582(7811), 230-233.

Kricorian, K., Civen, R., \& Equils, O. (2021). COVID-19 vaccine hesitancy: misinformation and perceptions of vaccine safety. Human Vaccines $\mathcal{E}$ Immunotherapeutics, 1-8.

Kwok, K. O., Lai, F., Wei, W. I., Wong, S. Y. S., \& Tang, J. W. (2020). Herd immunityestimating the level required to halt the COVID-19 epidemics in affected countries. Journal of Infection, 80(6), e32e33.

Loomba, S., de Figueiredo, A., Piatek, S. J., de Graaf, K., \& Larson, H. J. (2021). Measuring the impact of COVID-19 vaccine misinformation on vaccination intent in the UK and USA. Nature Human Behaviour, 5(3), 337-348.

Mahajan, R., Gupta, P. \& Kushwaha, P. (2022, in press). Role of digital misinformation in analyzing COVID 19 Vaccine Hesitancy. International Journal of Management Practice. (ahead-of-print).

Massey PM, Budenz A, Leader A, Fisher K, Klassen AC, Yom-Tov E. (2018). What drives health professionals to tweet about \#HPVvaccine? Identifying strategies for effective communication. Preventing Chronic Disease, Vol. 15, No. 26: https://doi.org/10.5888/pcd15.170320

McClure, C. C., Cataldi, J. R., \& O'Leary, S. T. (2017). Vaccine hesitancy: where we are and where we are going. Clinical therapeutics, 39(8), 1550-1562.
Meleo-Erwin Z, Basch C, MacLean SA, Scheibner C, Cadorett V (2017). To each his own: discussions of vaccine decisionmaking in top parenting blogs. Human Vaccine Immunotherapeutics, Vol. 13, No.8, pp.1895-901.

https://doi.org/10.1080/21645515.2017.1 $\underline{321182}$

Muric, G., Wu, Y., \& Ferrara, E. (2021). COVID-19 Vaccine Hesitancy on Social Media: Building a Public Twitter Dataset of Anti-vaccine Content, Vaccine Misinformation and Conspiracies. arXiv preprint arXiv:2105.05134.

Praveen, S. V., Ittamalla, R., \& Deepak, G. (2021). Analyzing Indian general public's perspective on anxiety, stress and trauma during Covid-19-a machine learning study of 840,000 tweets. Diabetes $\mathcal{E}$ Metabolic Syndrome: Clinical Research $\mathcal{E}$ Reviews, 15(3), 667-671.

Roozenbeek, J. (2020). Susceptibility to misinformation about COVID-19 376 around the world. Royal Society Open Science, 7(201199), 377. https://doi.org/10.1098/rsos.201199

Sanche, S., Lin, Y. T., Xu, C., Romero-Severson, E., Hengartner, N., \& Ke, R. (2020). High contagiousness and rapid spread of severe acute respiratory syndrome coronavirus 2. Emerging infectious diseases, 26(7), 1470.

Tregoning, J. S., Flight, K. E., Higham, S. L., Wang, Z., \& Pierce, B. F. (2021). Progress of the COVID-19 vaccine effort: viruses, vaccines and variants versus efficacy, effectiveness and escape. Nature Reviews Immunology, 1-11.

Welle D. (Jan 01, 2021). Anti-vaccine sentiment rife in Poland. The Indian Express, Available at https://indianexpress.com/article/worl $\mathrm{d} /$ anti-vaccine-sentiment-rife-in-poland7128797/ (Accessed 12 ${ }^{\text {th }}$ October 2021)

World Health Organization (March 11, 2020). WHO Director-General's opening remarks at the media briefing on COVID19-11 March 2020. Available on https://www.who.int/directorgeneral/speeches/detail/who-directorgeneral-s-opening-remarks-at-the-media- 
briefing-on-covid-19---11-march2020 (Accessed $12^{\text {th }}$ October 2021)

Sinnenberg L, Buttenheim AM, Padrez K, Mancheno C, Ungar L, Merchant RM. (2020). Twitter as a tool for health research: a systematic review. American journal of public health, Vol.107, No.1, pp. 8.

https://doi.org/10.2105/AJPH.2016.3035 $\underline{12}$

Quinn, S. C., Jamison, A. M., \& Freimuth, V. (2020). Communicating effectively about emergency use authorization and vaccines in the COVID-19 pandemic. American Journal of PublicHealth. https://ajph.aphapublications.org/doi/f $\underline{\text { ull/10.2105/AJPH.2020.306036 }}$
Bogart, L. M., Ojikutu, B. O., Tyagi, K., Klein, D. J., Mutchler, M. G., Dong, L., ... \& Kellman, S. (2021). COVID-19 related medical mistrust, health impacts, and potential vaccine hesitancy among Black Americans living with HIV. Journal of Acquired Immune Deficiency Syndromes, Vol. 86, No. 2, p. 200.

Fu C, Wei Z, Pei S, Li S, Sun X, Liu P. (2020). Acceptance and preference for COVID-19 vaccination in health-care workers (HCWs). medRxiv.

Thunstrom, L., Ashworth, M., Finnoff, D., \& Newbold, S. (2020). Hesitancy towards a COVID-19 vaccine and prospects for herd immunity. Available at SSRN 3593098. 